\title{
FURTHER STUDIES OF FULLY DEVELOPED FLOW THROUGH DUCTS OF ARBITRARY SECTION BY THE CONTOUR LINE-CONFORMAL MAPPING TECHNIQUE
}

\author{
J. MAZUMDAR \\ Department of Applied Mathematics \\ University of Adelaide \\ Australia, 5005 \\ and \\ D. HOPKINS \\ Department of Applied Mathematics \\ University of Adelaide \\ Australia, 5005
}

(Received December 19, 1995)

\begin{abstract}
The paper discusses fully developed flow of viscous fluid through ducts of arbitrary cross-section. The method uses a constant velocity contour line in a typical cross-section of the duch as an independent variable. The amplitude of the oscillatory velocity is then obtained from an ordinary integro-differential equation. Several examples of a practical nature are given, with some that have not yet been discussed in the literature. All details are explained by graphs.
\end{abstract}

KEY WORDS AND PHRASES: Duct flow, viscous fluids, velocity contours, conformal mappings. 1991 AMS SUBJECT CLASSIFICATION CODES: 76MXX, 65DXX.

\section{INTRODUCTION}

This paper is a further extension to previous work that has been done in the area of oscillatory fluid flow in ducts. In a previous paper [9], a method was proposed for the study of fully developed parallel flow of Newtonian viscous fluid in uniform straight ducsts of very general cross-section. The proposed method is based upon the concept of a family of contour-lines of constant velocity, $u(x, y)=$ const., in a typical cross-section of the duct and considering such line as an independent variable. Since the details of the method used in this study have been discussed by the first author in an earlier publication [9], only a brief discussion of the method is presented here.

According to the method, the governing equation for the axial velocity component $w(x, y, \tau)$ at any time $\tau$ is given by

$$
\frac{d^{2} W}{d u^{2}} \oint_{c_{u}} \sqrt{t} d s+\frac{d W}{d u} \oint_{c_{u}} \frac{\Delta^{2} u}{\sqrt{t}} d s-i \lambda^{2} W \oint_{c_{u}} \frac{d s}{\sqrt{t}}=\frac{1}{\mu} \frac{d P}{d z} \oint_{c_{u}} \frac{d s}{\sqrt{t}}
$$

where

$$
\begin{gathered}
w(x, y, \tau)=W(x, y) e^{i \omega \tau}, \quad p(z, \tau)=P(z) e^{i \omega \tau}, \\
t=u_{x}^{2}+u_{y}^{2}, \quad \lambda^{2}=\frac{\omega}{\nu} \quad \text { and } \quad \nu=\frac{\mu}{\rho} .
\end{gathered}
$$

The above equation is the integral form of the momentum equation for unsteady flow, ignoring any external forces. Here $\omega$ represents the frequency of oscillation, and $\lambda^{2}$ is a reduced frequency.

The family of contour lines of constant velocity, $C_{u}$, are represented by $u(x, y)=$ constant (see Fig. 1). If the exact equation for $u(x, y)$ is known, then the governing equation (1) yields the exact solution for the velocity component $w$. If, however, the exact form of $u(x, y)$ is not known a priori, then the method 


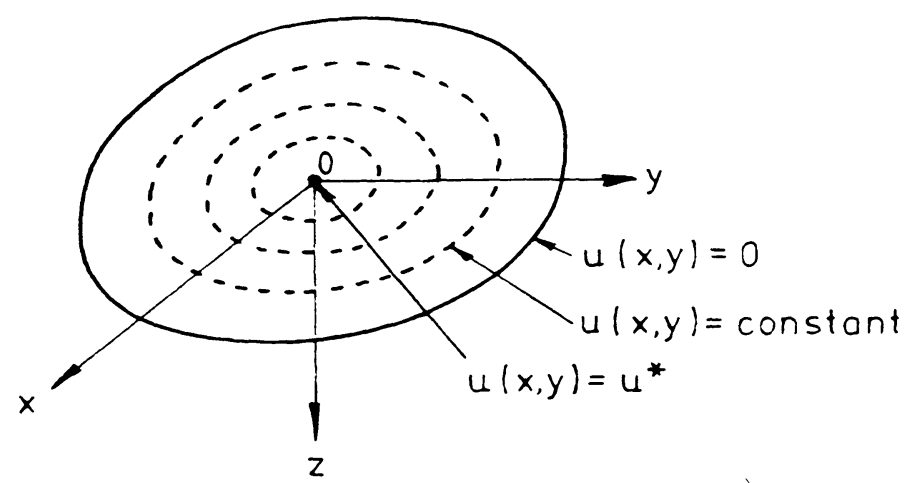

FIGURE I. Isovelocity contour lines.

of conformal transformation can be used to obtain an accurate approximate solution for the velocity distribution.

\section{APPLICATIONS}

\subsection{Solving the unsteady equation for a coaxial elliptical duct}

As shown in [9], considering the contour function as $u(x, y)=1-x^{2} / a^{2}-y^{2} / b^{2}$, the contour integrals in the unsteady equation can be solved easily, so that Eq. 1 becomes

$$
(1-u) \frac{d^{2} W}{d u^{2}}-\frac{d W}{d u}-\frac{i K^{2}}{4} W=\frac{K^{2}}{4 \mu \lambda^{2}} \frac{d P}{d z}
$$

where

$$
K^{2}=\frac{2 a^{2} b^{2} \lambda^{2}}{\left(a^{2}+b^{2}\right)}
$$

Introducing a new variable $f$ such that

$$
f^{2}=1-u
$$

Eq. 4 reduces to

$$
\frac{d^{2} W}{d f^{2}}+\frac{1}{f} \frac{d W}{d f}-i K^{2} W=\frac{K^{2}}{\mu \lambda^{2}} \frac{d P}{d z}
$$

which has a solution that is given by

$$
W=A_{1} I_{0}(\sqrt{i} K f)+A_{2} K_{0}(\sqrt{i} K f)+\frac{i}{\mu \lambda^{2}} \frac{d P}{d z}
$$

where $A_{1}$ and $A_{2}$ are arbitrary constants and $I_{0}$ and $K_{0}$ are modified Bessel functions of the first and second kind. The constants $A_{1}$ and $A_{2}$ are found using the viscous no-slip boundary conditions that occur at duct walls.

If the duct is simply connected, then the boundary values of $u$ are

$$
u=0 \text { and } u=u^{*}=1
$$

at the outer boundary and the origin, respectively. This produces the corresponding boundary conditions

$$
\left.W(f)\right|_{f=1}=0 \text { and }\left.W(f)\right|_{f=0}=0
$$

and so solving Eq. 8 for $A_{1}$ and $A_{2}$ we get

and

$$
A_{1}=\frac{-i}{\mu \lambda^{2}} \frac{d P}{d z} \frac{1}{I_{0}(\sqrt{i} K)}
$$

$$
A_{2}=0
$$

and thus, Eq. 8 finally gives 


$$
W=\frac{\imath}{\mu \lambda^{2}} \frac{d P}{d z}\left[1-\frac{I_{0}(\sqrt{i} K f)}{I_{0}\left(\sqrt{i} K^{\prime}\right)}\right]
$$

If we make the substitution

$$
I_{0}(\sqrt{\imath} x)=b e r x+i b e \imath x
$$

then $W$ can be given in the form

$$
\begin{aligned}
W & =W_{1}+\imath W_{2} \\
& =\sqrt{W_{1}^{2}+W_{2}^{2}} e^{\imath \delta}, \quad \delta=\tan ^{-1}\left(\frac{W_{2}}{W_{1}}\right)
\end{aligned}
$$

where $\delta$ denotes the phase difference between pressure and velocity.

We thus have

$$
W_{1}=\frac{1}{\mu \lambda^{2}} \frac{d P}{d z}\left(\frac{\text { ber Kbei } K f-b e i K b e r K f}{b e r^{2} K+b e i^{2} K^{\prime}}\right)
$$

and

$$
W_{2}=\frac{1}{\mu \lambda^{2}} \frac{d P}{d z}\left(1-\frac{b e r K b e r K f+b e i K b e \imath K f}{b e r^{2} K+b e i^{2} K}\right) .
$$

The amplitude velocity, $V$, is given by

$$
V=\sqrt{W_{1}^{2}+W_{2}^{2}}
$$

so

$$
V=\frac{1}{\mu \lambda^{2}} \frac{d P}{d z} \frac{\left[\begin{array}{l}
b e r^{2} K(b e r K-b e r K f)^{2}+3 b e r^{2} K b e i K \\
-2 b e \imath K b e i K f b e r^{2} K+b e i^{2} K(b e \imath K-b e \imath K f)^{2} \\
+b e i^{2} K b e r^{2} K f-2 b e i^{2} K b e r K b e r K f
\end{array}\right]^{\frac{1}{2}}}{\left(b e r^{2} K+b e i^{2} K\right)} .
$$

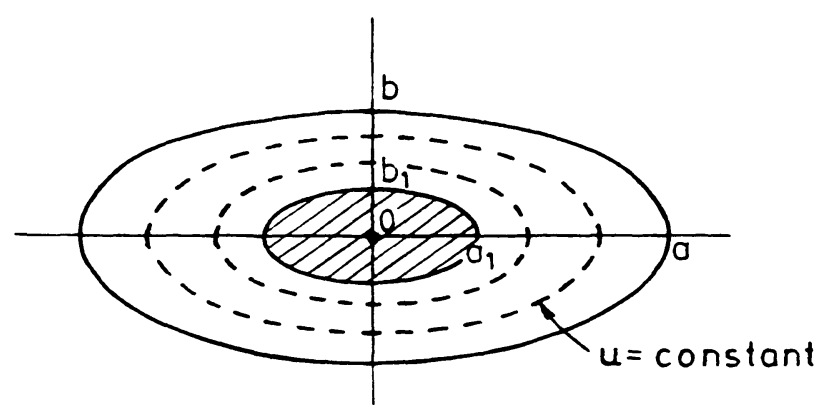

FIGURE 2. Cross-section of a doubly connected ellipse.

If, however, the duct is doubly connected (see Fig. 2), then the boundary values for $u$ are

$$
u=0 \text { and } u=u^{*}=1-\beta^{2}
$$

on the outer and inner walls, respectively, where $\beta$ is the similarity condition between the duct walls, ie.,

$$
\beta=\frac{a_{1}}{a}=\frac{b_{1}}{b}, \quad 0<\beta<1 .
$$

The corresponding boundary conditions are then

$$
\left.W(f)\right|_{f=1}=0 \text { and }\left.W(f)\right|_{f=\beta}=0
$$

Substituting these conditions into Eq. 8 we get

$$
\begin{aligned}
& A_{1}=\frac{i}{\mu \lambda^{2}} \frac{d P}{d z}\left[\frac{K_{0}(\sqrt{i} K)-K_{0}(\sqrt{i} K \beta)}{K_{0}(\sqrt{i} K \beta) I_{0}(\sqrt{i} K)-K_{0}(\sqrt{i} K) I_{0}(\sqrt{i} K \beta)}\right], \\
& A_{2}=\frac{i}{\mu \lambda^{2}} \frac{d P}{d z}\left[\frac{I_{0}(\sqrt{i} K \beta)-I_{0}\left(\sqrt{i} K^{\prime}\right)}{K_{0}(\sqrt{i} K \beta) I_{0}\left(\sqrt{i} K^{\prime}\right)-K_{0}(\sqrt{i} K) I_{0}\left(\sqrt{i} K^{\prime} \beta\right)}\right]
\end{aligned}
$$



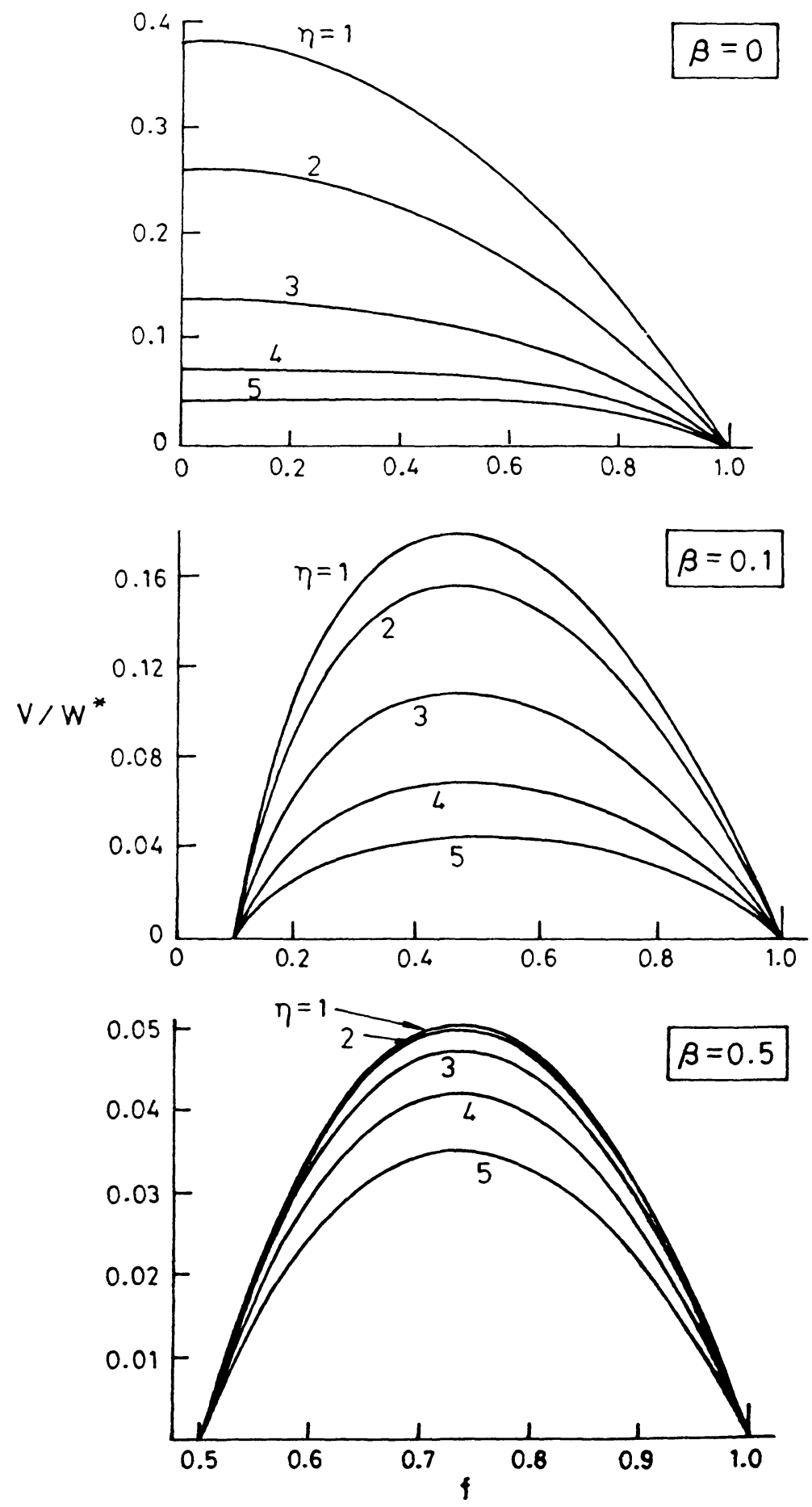

FIGURE 3. Amplitude distribution of oscillatory flow over the cross-section of a coaxial elliptical duct for $\beta=0.0,0.1,0.5, \eta=1,2,3,4,5$ and $f=0.0, \ldots, 1.0$. 

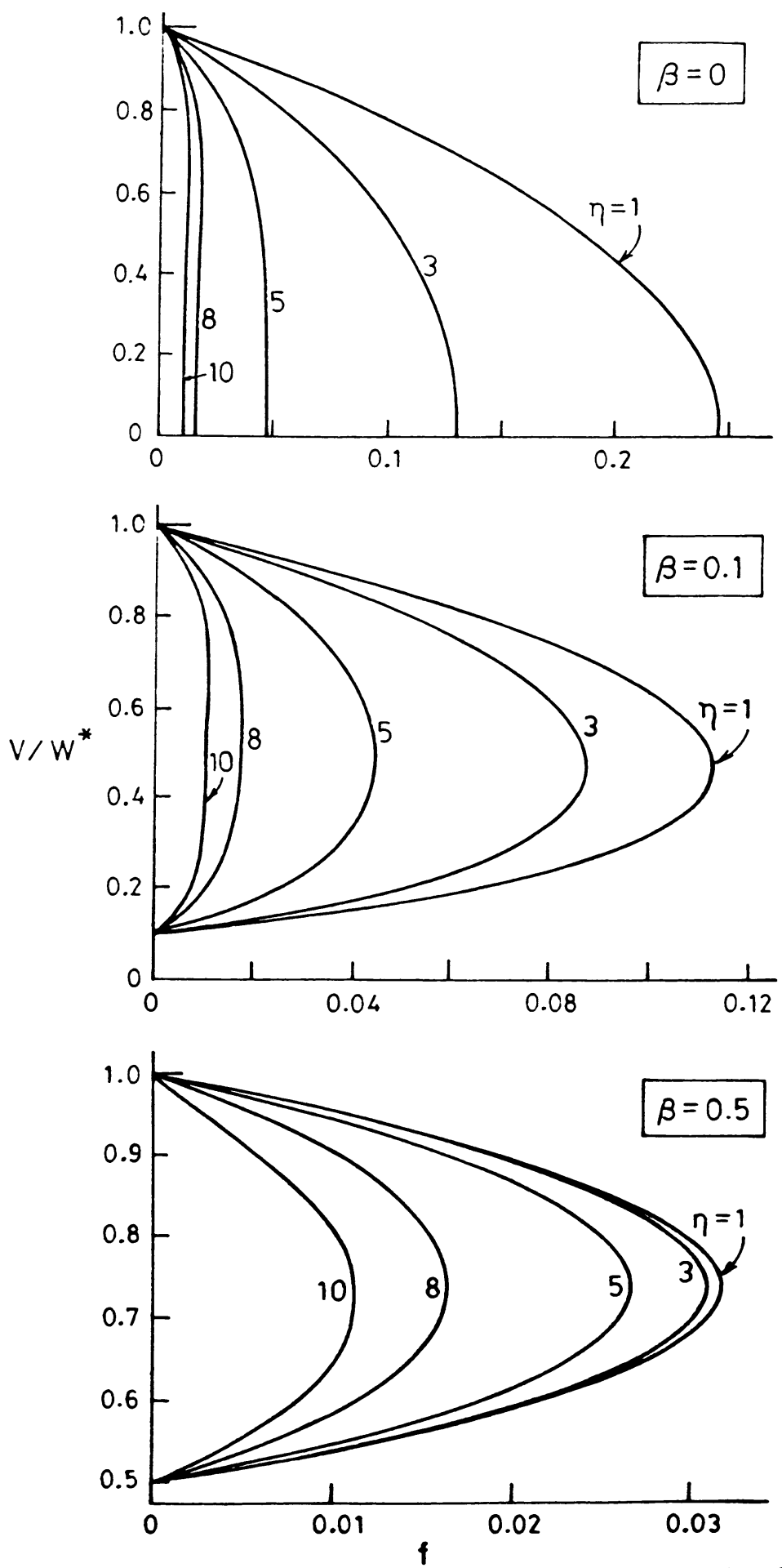

FIGURE 4. Amplitude distribution of oscillatory flow over the cross-section of an annular duct for $\beta=0.0,0.1,0.5, \eta=1,3,5,8,10$ and $f=0.0, \ldots, 1.0$. 
and thus Eq. 8 finally becomes

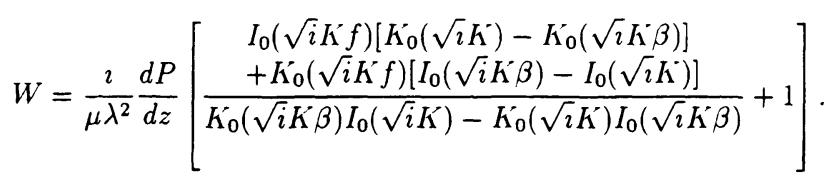

The amplitude velocity, $V$, in this case, then becomes

$$
V=\frac{1}{\mu \lambda^{2}} \frac{d P}{d z}\left[\begin{array}{c}
{\left[L_{r}\left(f K, \beta K^{\prime}\right)-L_{r}\left(f K^{\prime}, K^{\prime}\right)-L_{r}\left(K, \beta K^{\prime}\right)\right]^{2}} \\
\frac{+\left[L_{\imath}\left(f K, \beta K^{\prime}\right)-L_{\imath}\left(f K^{\prime}, K^{\prime}\right)-L_{\imath}\left(K, \beta K^{\prime}\right)\right]^{2}}{L_{r}^{2}\left(K^{\prime}, \beta K^{\prime}\right)+L_{\imath}^{2}\left(K, \beta K^{\prime}\right)}
\end{array}\right]^{\frac{1}{2}}
$$

where $L(\sigma, \tau)$ is a new function of two independant variables $\sigma$ and $\tau$ given by [11]

$$
\begin{aligned}
L(\sigma, \tau) & =L_{r}(\sigma, \tau)+i L_{\imath}(\sigma, \tau) \\
& =I_{0}(\sigma \sqrt{i}) K_{0}(\tau \sqrt{i})-I_{0}(\tau \sqrt{i}) K_{0}(\sigma \sqrt{i}) .
\end{aligned}
$$

and

$$
\begin{aligned}
& L_{r}(\sigma, \tau)=b e r \sigma k e r \tau-k e r \sigma b e r \tau-b e i \sigma k e i \tau+k e i \sigma b e i \tau \\
& L_{\imath}(\sigma, \tau)=b e i \sigma k e r \tau-k e i \sigma b e r \tau+b e r \sigma k e i \tau-k e r \sigma b e i \tau
\end{aligned}
$$

$b e r, b e \imath$ and $k e r, k e i$ are the real and imaginary parts of $I_{0}$ and $K_{0}$ respectively. The value, $V$, thus obtained will give the amplitude distribution of the velocity in the doubly connected elliptical cross-section of a duct.

Since according to the authors' knowledge there is no published results for this problem we will for the sake of comparison and confirmation of our approach, consider the limiting case when two ellipses become two circles. It is interesting to note that if one puts $a=b$ and $a_{1}=b_{1}$ so that the two ellipses reduce to two circles, then the results obtained for this case coincide with the results given in [11] for the case of an annular duct. We further note that in Eq. 7, the only quantity affected by this substitution is the parameter $K^{*}$. Fig. 3 shows the graphs of $V / W^{*}$ for a coaxial elliptical duct, where $W^{*}=\left|\frac{b^{2}}{\mu} \frac{d P}{d z}\right|$, and for aspect ratio $a / b=2$, and for three different values of $\beta(0.0,0.1,0.5)$ and various values of the parameter $\eta=b \lambda$. In Fig. 4 the graphs of $V / W^{*}$ are shown with $a / b=1, \beta(0.0,0.1,0.5)$ and a set of values of $\eta$. The results in Fig. 4 agree precisely with those of Tsangaris [11]. It is further interesting to note that the graph in Fig. 3 corresponding to $\beta=0$ coincides with the graph for an elliptical duct given in [9].

\subsection{Conformal mapping technique}

For more complicated cross-sections an exact equation for the isovelocity contours may not exist, so conformal mapping can be used (see Fig. 5). This assumes a one-to-one correspondance between the $U(\xi, \eta)=$ constant contours and the $u(x, y)=$ constant contours where $z=x+i y$ and $\zeta=\xi+\imath \eta$. Therefore as long as a function exists to map the cross-section onto the unit circle, it is not necessary to know $u$. The function $U$ is represented as

$$
U(\xi, \eta)=1-\xi^{2}-\eta^{2}
$$

and the contour integrals appearing in the unsteady equation (Eq. 1) are

$$
\begin{aligned}
\oint_{C_{u}} \sqrt{t} d s & =2 \oint_{C_{U}} \sqrt{U_{\zeta} U_{\zeta}} d S=4 \pi(1-U), \\
\oint_{c_{u}} \frac{\nabla^{2} u}{\sqrt{t}} d s & =2 \oint_{c_{U}} \frac{U_{\bar{\zeta}}}{\sqrt{U_{\zeta} U_{\bar{\zeta}}}} d S=-4 \pi, \\
\oint_{c_{u}} \frac{d s}{\sqrt{t}} & =\oint_{c_{U}} \frac{1}{2 \sqrt{U_{\zeta} U_{\bar{\zeta}}}}\left|\frac{d z}{d \zeta}\right|^{2} d S=\frac{1}{2 \sqrt{1-U}} \oint_{c_{U}}\left|\frac{d z}{d \zeta}\right|^{2} d S
\end{aligned}
$$

where

$$
t=u_{x}^{2}+u_{y}^{2}=4 U_{\zeta} U_{\bar{\zeta}}\left|\frac{d z}{d \zeta}\right|^{2}
$$



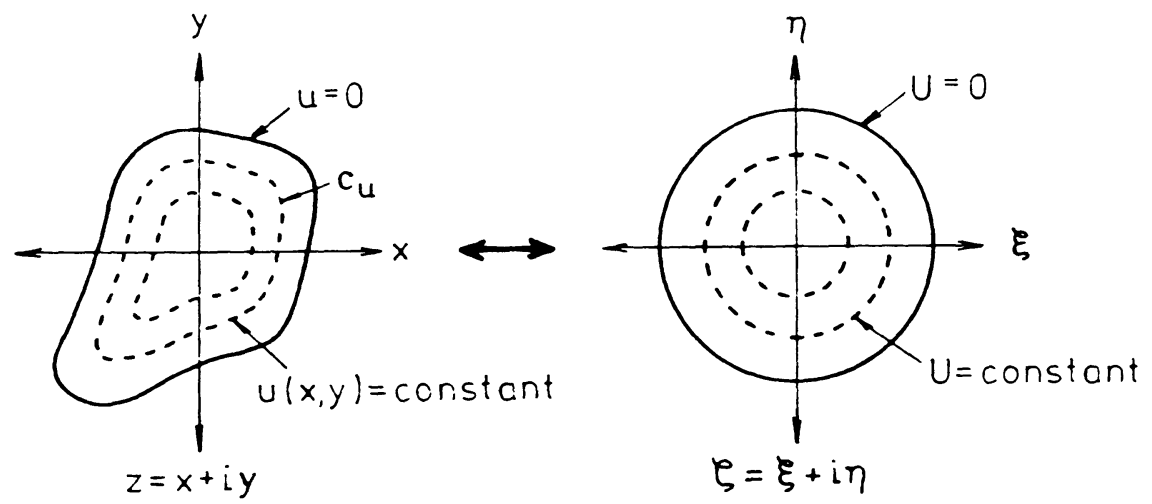

So Eq. 1 becomes

FiGURE 5. Conformal mapping geometry

$$
(1-U) \frac{d^{2} W}{d U^{2}}-\frac{d W}{d U}-i H(U) W=\frac{H(U)}{\mu \lambda^{2}} \frac{d P}{d z}
$$

where

$$
H(U)=\frac{\lambda^{2} \oint_{c_{U}}\left|\frac{d z}{d \zeta}\right|^{2} d S}{8 \pi \sqrt{1-U}}
$$

and $z=\psi(\zeta)$ is the mapping function for the cross-section.

If we again introduce a variable, $F$, such that

$$
F^{2}=1-U
$$

then substitution into Eq. 34 produces

$$
\frac{d^{2} W}{d F^{2}}+\frac{1}{F} \frac{d W}{d F}-i H^{\prime}(F) W=\frac{H^{\prime}(F)}{\mu \lambda^{2}} \frac{d P}{d z}
$$

where

We note that

$$
H^{\prime}(F)=\frac{\lambda^{2} \oint_{c_{U}}\left|\frac{d z}{d \zeta}\right|^{2} d S}{2 \pi F}
$$

$$
H^{\prime}(F)=\frac{\lambda^{2} \int_{0}^{2 \pi}\left|\frac{d z}{d \zeta}\right|^{2} r d \theta}{2 \pi F}=\lambda^{2}\left|\frac{d z}{d \zeta}\right|^{2}=\alpha^{2} \quad \text { (say). }
$$

where $\alpha^{2}$ depends on the complex variable $\zeta$. If we make the substitution $H^{\prime}(F)=\alpha^{2}$ in Eq. 37, then Eq. 37 will become identical to Eq. 7 where instead of $K^{2}$ we have $\alpha^{2}$, and instead of variable $f$ we have $F$. We therefore note that Eqn 24 gives the solution for any duct of arbitrary cross-section with a value of $K$; depending on the geometry of the duct boundary. This also applies for the complex mapping technique approach if we replace the parameter $K^{2}$ by $\alpha^{2}$.

\section{ILLUSTRATIONS}

3.1. Simply connected cross-sectional areas

(a) Flow through a duct of semi-elliptical cross-section

Consider the case of flow through a semi-elliptical duct (Fig. 6). A complicated conformal mapping is used to get the mapping function of this shape. First we have to map the unit circle onto a semi-circle of radius $r$ using [10]

$$
z=r \frac{t-1}{t+1}, \quad t^{2}=\frac{1}{\imath}\left(\frac{\zeta+1}{\zeta-1}\right)
$$

then we map the semi-circle onto a semi-ellipse using the same function that maps the unit circle onto an ellipse, given by [10] 
where

$$
\begin{aligned}
z= & \left(1-\frac{r^{2}}{8}+\frac{3 r^{4}}{128}\right)\left[\zeta+\left(\frac{r}{2}-\frac{r^{3}}{4}+\frac{3 r^{5}}{32}\right) \zeta^{3}+\left(\frac{r^{2}}{2}-\frac{9 r^{4}}{16}\right) \zeta^{5}\right. \\
& \left.+\left(\frac{5 r^{3}}{8}-\frac{9 r^{5}}{8}\right) \zeta^{7}+\frac{7 r^{4} \zeta^{9}}{8}+\frac{21 r^{5} \zeta^{11}}{16}\right]
\end{aligned}
$$

$$
r=\frac{\delta^{2}-1}{\delta^{2}+1}, \quad \delta=\frac{a}{b}>1 .
$$

Substitution of $\alpha=\left|\frac{d z}{d \zeta}\right|$ into Eq. 18 will then give the appropriate velocity amplitude. The graphs for $V / W^{*}$ are given in Fig. 7 with $a / b=2$ and various values of $\eta=a \lambda$, where $W^{*}=\left|\frac{a^{2}}{\mu} \frac{d P}{d z}\right|$.

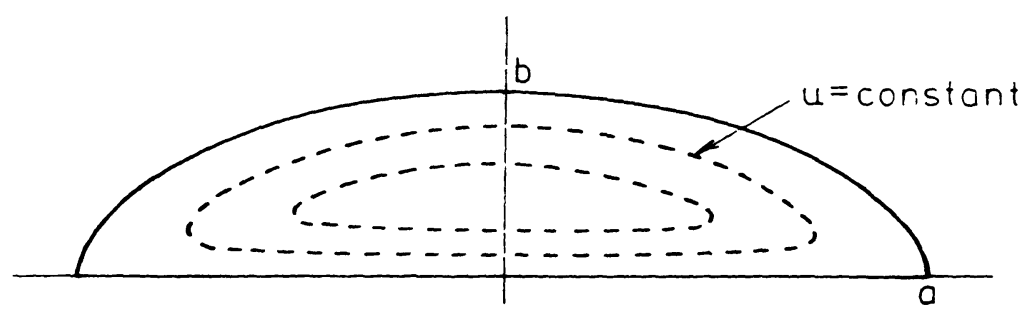

FIgURE 6. Cross-section of a semi-ellipse.

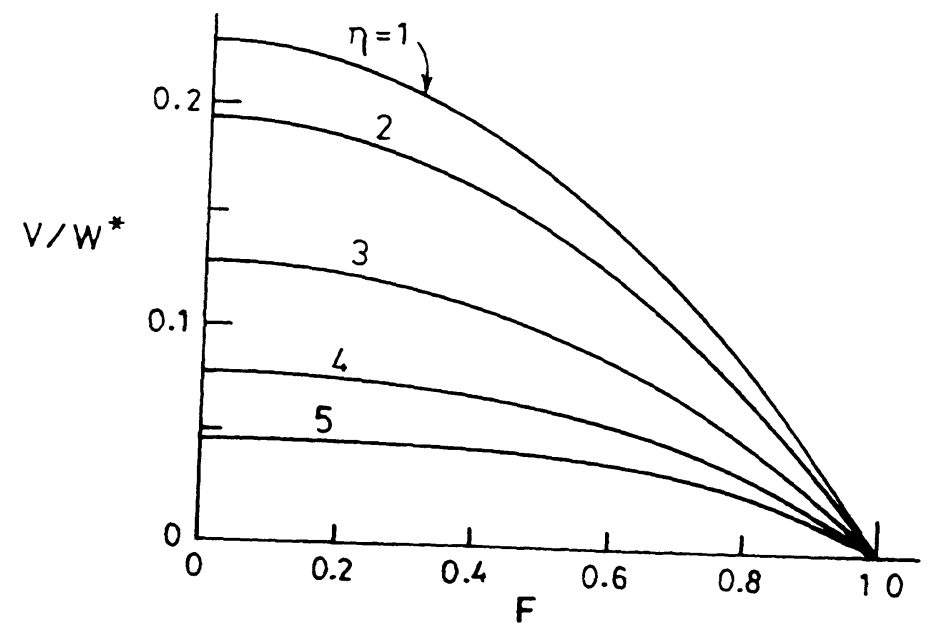

FIGURE 7. Amplitude distribution of oscillatory flow over the cross-section of a simply connected semi-elliptical pipe for $\eta=1,2,3,4,5, \zeta=0.1$ and $F=0.1, \ldots, 1.0$.

(b) Flow through a simply connected square duct with rounded comers

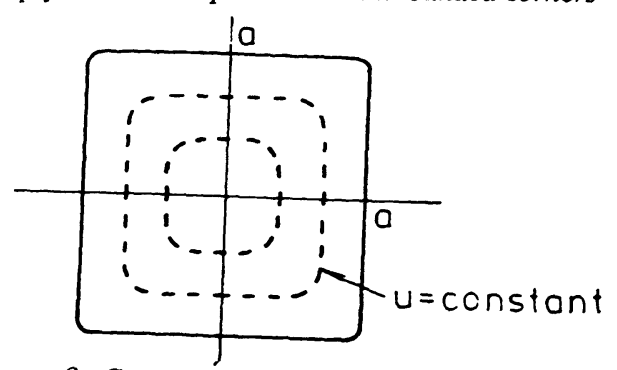

FIGURE 8. Cross-section of a square duct with rounded corners. 
Consider another example of a square duct with rounded corners (Fig. 8). The cross-section for this shape also has a mapping function given by [10]

$$
z=\frac{25}{24} a\left(\zeta-\frac{1}{25} \zeta^{2}\right)
$$

where $2 a$ is the length of the sides of the square. Hence, the quantity $\alpha$ becomes

$$
\alpha=\frac{625}{576} a^{2}\left|1-\frac{2}{25} \zeta\right|^{2}
$$

and again, by replacing $K$ by $\alpha$ in Eq. 18 we get the solutions for $V / W^{*}$ displayed in Fig. 9 for side length $2 a=$ and a set of values $\eta=a \lambda$, where $W^{*}=\left|\frac{a^{2}}{\mu} \frac{d P}{d z}\right|$.

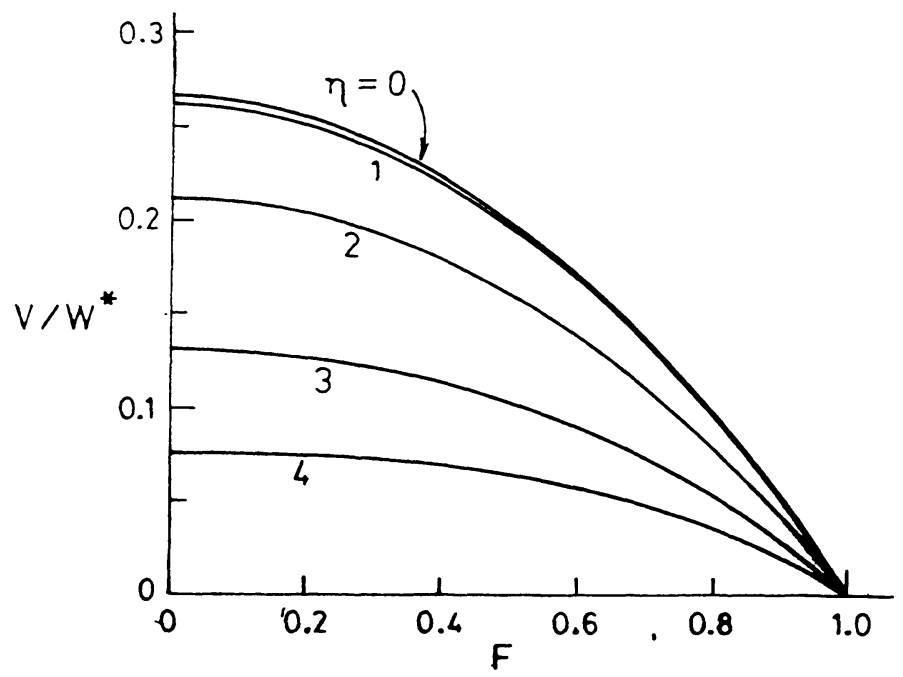

FIGURE 9. Amplitude distribution of the oscillatory flow over the cross-section of a simply connected square with rounded corners for $\eta=0,1,2,3,4, \zeta=0.1$ and $F=0.0, \ldots, 1.0$.

3.2. Doubly connected cross-sectional areas

(a) Flow through a doubly connected duct of square cross section

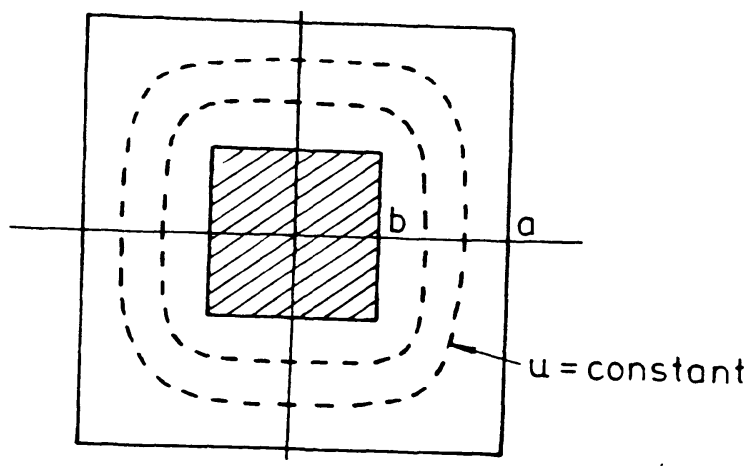

FIGURE 10. Cross-section of a doubly connected square.

First consider the case when both inner and outer boundaries have polygonal cross-section. Therefore we want to map this shape onto a doubly connected annular region where the outer boundary is the unit circle (see Fig. 10). We do this using the well known Schwartz-Christoffel transformation to approximate the mapping function as it is not known [3].

$$
z=\psi(\zeta)=a_{1} \Gamma_{n} \int_{0}^{\zeta}\left(1+t^{n}\right)^{-\frac{2}{n}} d t
$$

where $a_{1}=$ the apothem of the regular polygon,

$n=$ the number of sides on the polygon, and

$\Gamma_{n}$ is the mapping coefficient given by 


$$
\Gamma_{n}=1 / \int_{0}^{1}\left(1+t^{n}\right)^{-\frac{2}{n}} d t
$$

The numerical values of the mapping coefficients for some regular polygons are given in Table 1 ,

TABLE I. Numerical values for mapping coefficients of regular polygonal transformations.

\begin{tabular}{|c|c|}
\hline \# Sides, $n$ & Mapping Coefficient, $\Gamma_{n}$ \\
\hline 3 (triangle) & 1.135 \\
4 (square) & 1.079 \\
5 (pentagon) & 1.052 \\
6 (hexagon) & 1.038 \\
7 (heptagon) & 1.028 \\
8 (octagon) & 1.022 \\
\hline
\end{tabular}

and from other results in the literature [3] we know that for a polygon of 4 sides, the mapping function can be approximated by

$$
z \approx a\left[1.0807 \zeta-0.1081 \zeta^{5}+0.045 \zeta^{9}-0.0242 \zeta^{13}+0.0174 \zeta^{17}-0.0126 \zeta^{21}\right] .
$$

This function can be used to map the unit circle onto the outer boundary square. Now we need to map another concentric circle of radius, $\gamma<1$, onto the inner boundary square. The value of the side length, $b$, in this square can be denoted by

$$
b=a \Gamma_{4} \int_{0}^{\gamma}\left(1-t^{4}\right)^{-\frac{1}{2}} d t
$$

so

$$
0.0126 \gamma^{21}-0.017 \gamma^{17}+0.0242 \gamma^{13}-0.045 \gamma^{9}+0.1081 \gamma^{5}-1.0807 \gamma+\frac{b}{a}=0
$$

must be solved to obtain the concentric inner circle radius, $\gamma$. We can then completely map this crosssection using the mapping function (Eq. 48) to obtain $H^{\prime}(F)$ after integrating across the contour integrals from 0 to $\sqrt{1-\gamma^{2}}$. The unsteady equation can then be solved, and Fig. 11 shows the graphs of $V / W^{*}$ for varying side ratios $\beta=\gamma / 1<1$ and values of $\eta=a \lambda$, where $W^{*}=\left|\frac{a^{2}}{\mu} \frac{d P}{d z}\right|$. The results in Fig. 11(a) for the limiting case, $\beta=0$ are also in excellent agreement with the results for a simply connected four-sided polygon given in [9].

(b) Flow through a duct of doubly connected cross-section with a polygonal outer boundary and a circular inner boundary

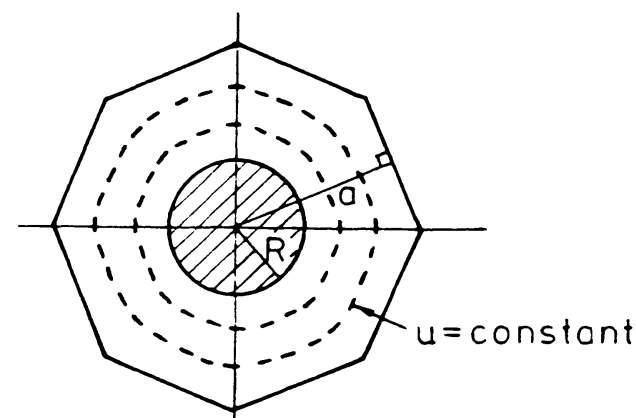

FIGURE 12. Cross-section of a doubly connected duct with polygonal outer boundary and circular inner boundary.

In this case we again want to transform the cross-section (see Fig. 12) onto an annular region. We know the mapping function for the outer boundary can be approximated by

$$
z=a\left[1.0219 \zeta-0.0282 \zeta^{9}+0.0092 \zeta^{19}-0.0048 \zeta^{28}-0.0008 \zeta^{37}+0.0026 \zeta^{41}\right] .
$$



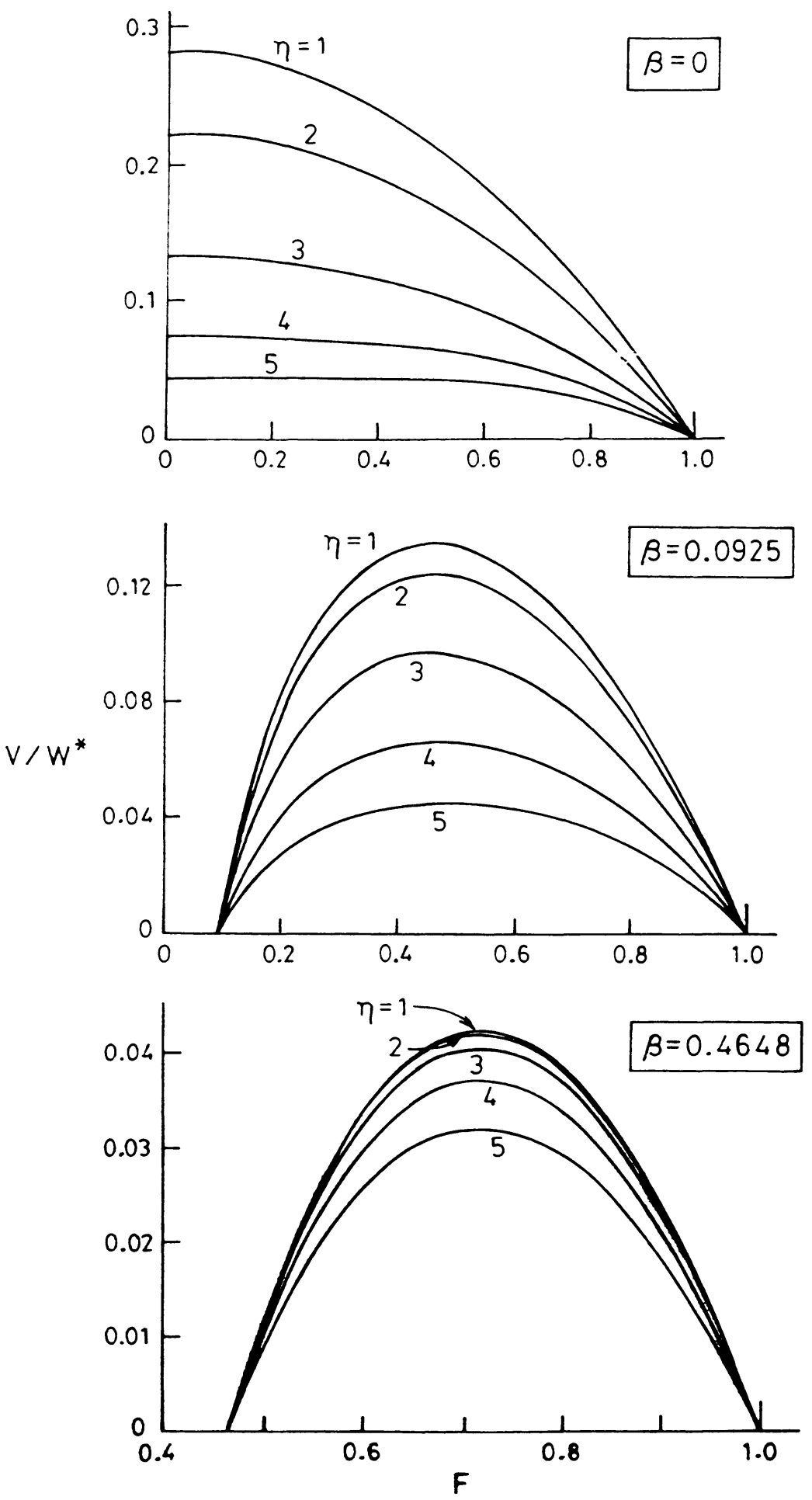

FIGURE I I. Amplitude sitribution of the oscillatory flow over the cross-section of a doublyconnected square for $\beta=0.0,0.0925,0.4648, \eta=1,2,3,4,5, \zeta=0.1$ and $F=$ $0.0, \ldots, 1.0$. 

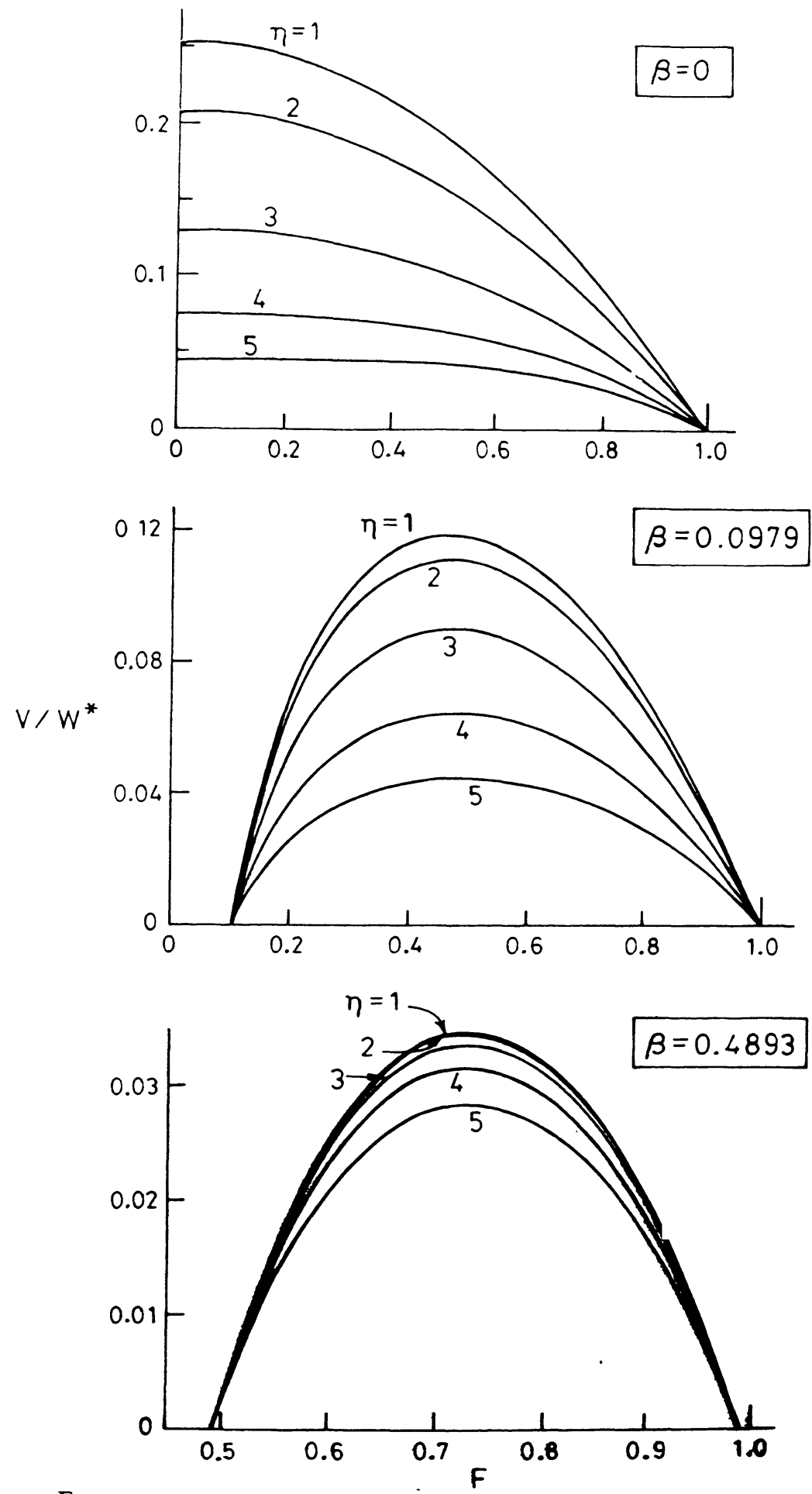

FIGURE I3. Amplitude distribution of the oscillatory flow over the cross-section of the doubly connected duct with polygonal outer boundary and inner boundary for $\beta=0.0,0.09786,0.48928, \eta=1,2,3,4,5, \zeta=0.1$ and $F=0.0, \ldots, 1.0$. 
We also know from Laura [7] that circles in the $\zeta$-plane will map into approximate circles in the $z$-plane, with radius of the inner boundary given by

$$
\beta=\frac{R}{c_{1}}
$$

where $c_{1}$ is the first coefficient of the mapping polynomial, and $R$ is the radius of the inner boundary in the physical plane. So

$$
\beta=\frac{R}{1.0219 a}
$$

and similarly to part (a) we integrate across the contour integrals from 0 to $\sqrt{1-\beta^{2}}$ and then use the mapping function, $z$, to solve the unsteady equation and obtain the graphs of $V / W^{*}$ displayed in Fig. 13.

(c) Flow through a duct of doubly connected cross-section with a corrugated outer boundary and a circular inner boundary

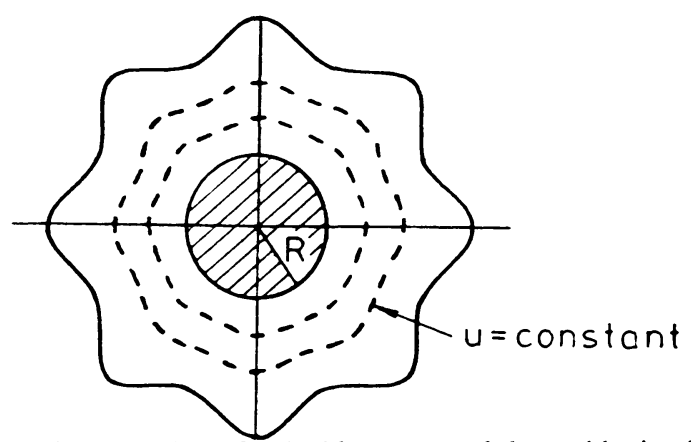

FiGURE I4. Cross-section of a doubly connected duct with circular inner boundary and corrugated outer boundary.

Consider the diagram in Fig. 14. To solve this case we adopt the same method as in part (b), except the mdpping function for a corrugated boundary is

$$
z=\frac{a}{1+m}\left(\zeta+m \zeta^{n+1}\right)
$$

where $n=$ the number of axes of symmetry,

$a=$ the radius of the circumscribing circle, and

$$
m \leq \frac{1}{n+1}
$$

and the radius of the inner concentric circle will be

$$
\beta=\frac{R(1+m)}{a} .
$$

The results are given in Fig. 15. 

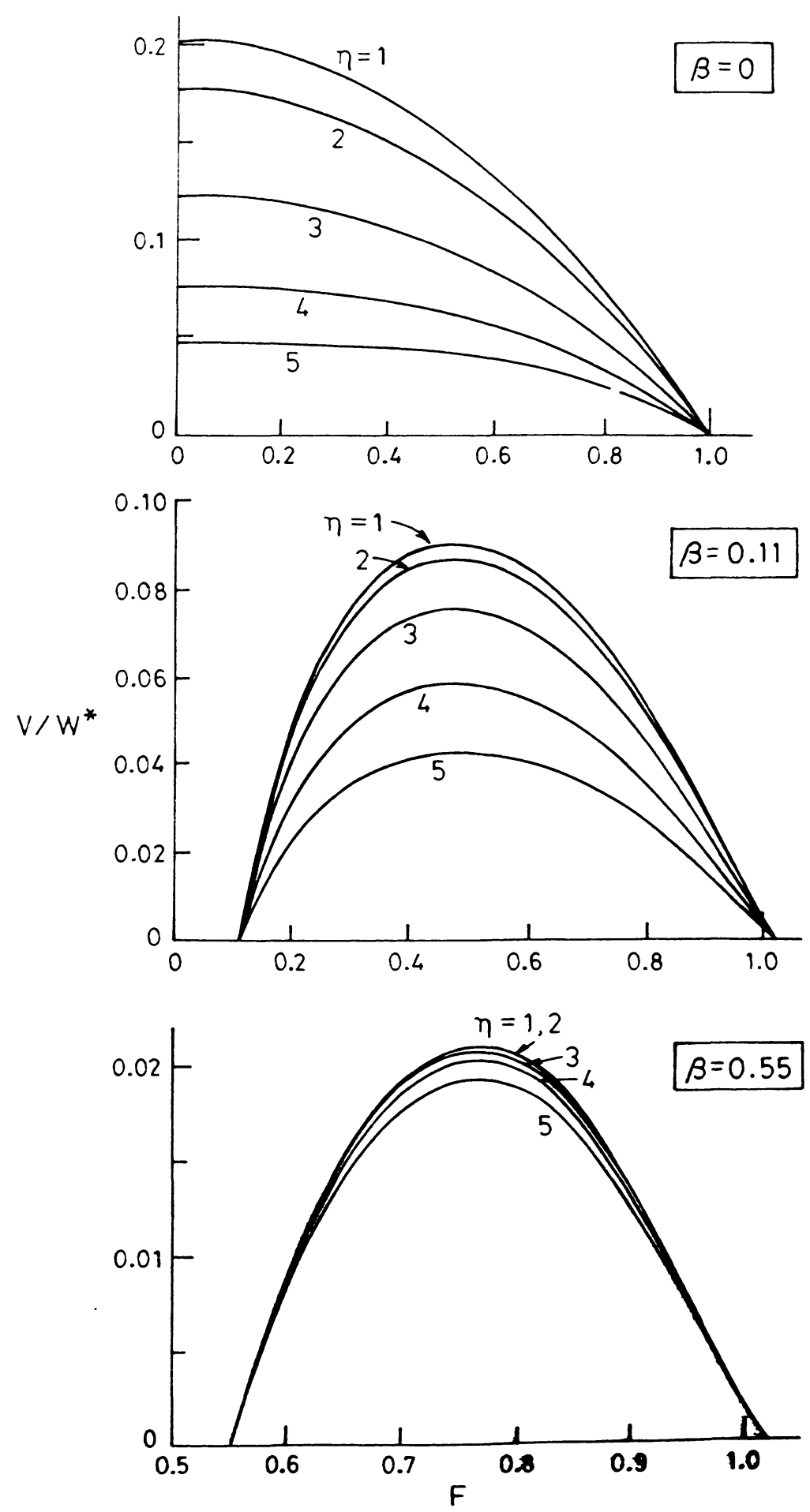

FIGURE 15. Amplitude distribution of the oscillatory flow over the cross-section of the doubly connected duct with circular inner boundary and corrugated outer boundary for $\beta=0.0,0.11,0.55, \eta=1,2,3,4,5, \zeta=0.1$ and $F=0.0, \ldots, 1.0$. 


\section{CONCLUSION}

We have thus obtained an accurate approximate solution for the analysis of a wide spectrum of fully developed fluid flow problems in simply and doubly-connected ducts of arbitrary cross-sections. The essence of the present approach is to reduce the partial differential equation for the transverse velocity component to an ordinary second order differential equation using the concept of contour lines on a typical cross-section of the duct. Next, the method of conformal transformation is applied to find the velocity distribution in a typical cross-section for a number of duct shapes. The approach presented is quite simple and straightforward.

The examples discussed show a very good agreement between the calculated values and the values existing in the literature, and confirm the usefulness of the method.

Furthermore, the present study has been limited to the study of velocity distribution in a duct flow. Many other problems, for example the dynamic response of MHD (magnetohydrodynamic) flow in conduits when subjected to pressure gradient can be analysed in a like manner, which is to be carried out in subsequent papers in this series.

ACKNOWLEDGEMENT. The present study has been sponsored by a grant from the Adelaide University Research Grant for which the first author wishes to gratefully acknowledge.

\section{REFERENCES}

[1] Casarella M.J., Laura P.J. and Chi M.: On the approximate solution of flow and heat transfer through non-circular conduits with uniform wall temperature. Brit. Journal App. Phys. 18 (1967) 1327-1335.

[2] Casarella M.J., Laura P.J. and Ferragut N.: On the approximate solution of flow and heat transfer through non-circular conduits with uniform wall temperature and heat generation. Nuc. Eng. and Des. 16 (1971) 387-398.

[3] Kantorovich L.V. and Krylov V.I.: Approximate Methods of Higher Analysis. The Netherlands: Gronigen (1958).

[4] Kober H.: Dictionary of Conformal Representations. Dover Publications inc. (1952).

[5] Laura P.A. and Ercoli R.: Analysis of unsteady MHD flow in conduits of arbitrary cross-section by a complex variable approach. J. Acoust. Soc. Amer. 51 (1972) 820-824.

[6] Laura P.A.A. and Faulstich Jr. A.J.: An application of conformal mapping to the determination of the natural frequency of membranes of regular polygonal shape. 9th Midwestern Conference, Madison, Wisconsin (1965) 155-60.

[7] Laura P.A., Romanelli E. and Maurizi M.J.: On the analysis of waveguides of doubly connected cross-section by the method of conformal mapping. J. Sound and Vib. 20 (1) (1972) 27-38.

[8] Mazumdar J.: A method for the study of transient heat conduction in plates of arbitrary cross-section. Nuc. Eng. and Des. 31 (1974) 383-390.

[9] Mazumdar J. and Dubey R.N.: A method for the study of fully developed parallel flow in straight ducts of arbitrary cross-section. J. Aust. Math. Soc., Ser. B 33 (1991) 211-239.

[10] Mazumdar J. and Hill D.: A note on the determination of cutoff frequencies of hollow waveguides by a contour line-conformal mapping technique. J. App. Acoust. 21 (1987) 23-37.

[11] Tsangaris S.: Oscillatory flow of an incompressible viscous fluid in a straight annular pipe. J. de Mécanique Théorique et Appliqué 3 (1984) 467-478. 


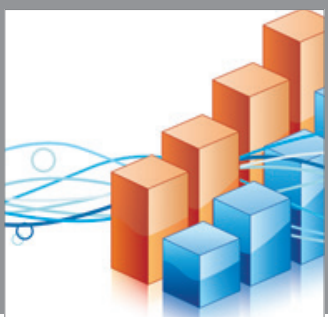

Advances in

Operations Research

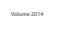

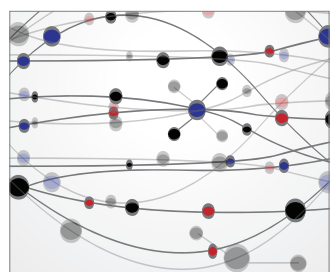

\section{The Scientific} World Journal
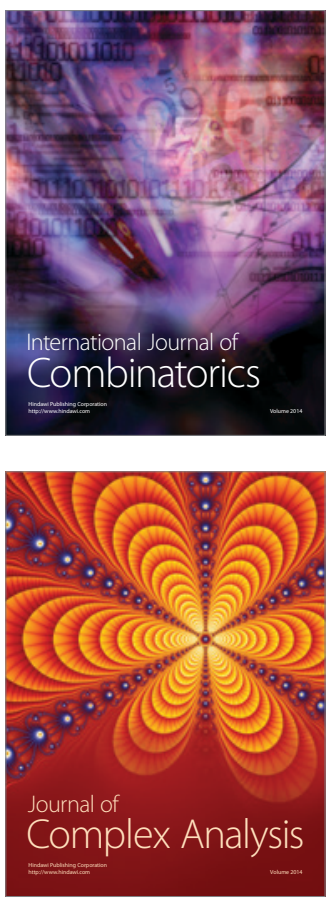

International Journal of

Mathematics and

Mathematical

Sciences
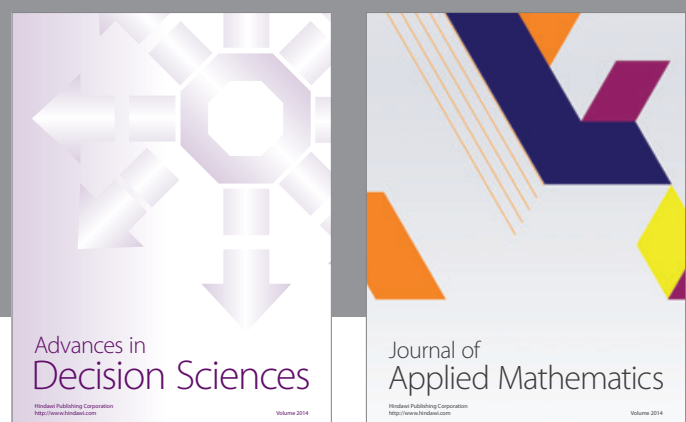

Journal of

Applied Mathematics
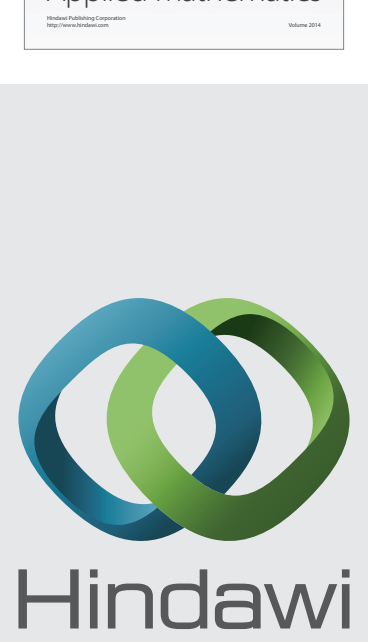

Submit your manuscripts at http://www.hindawi.com
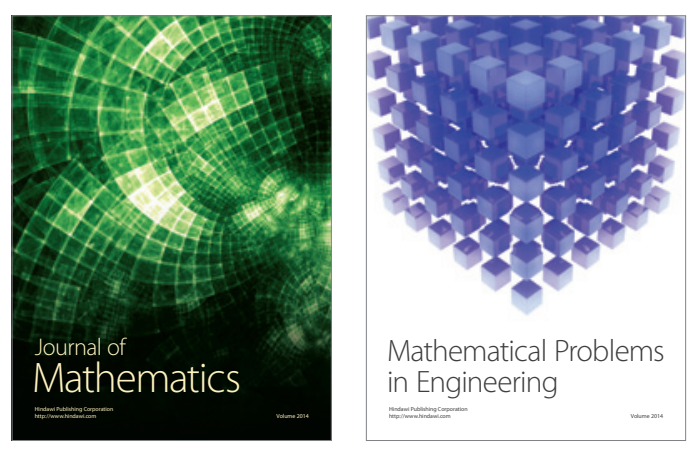

Mathematical Problems in Engineering
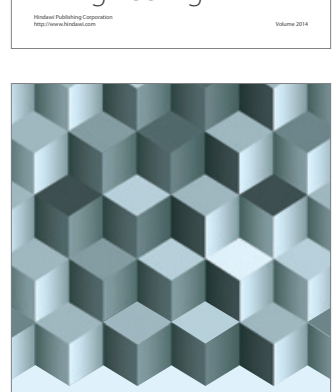

Journal of

Function Spaces
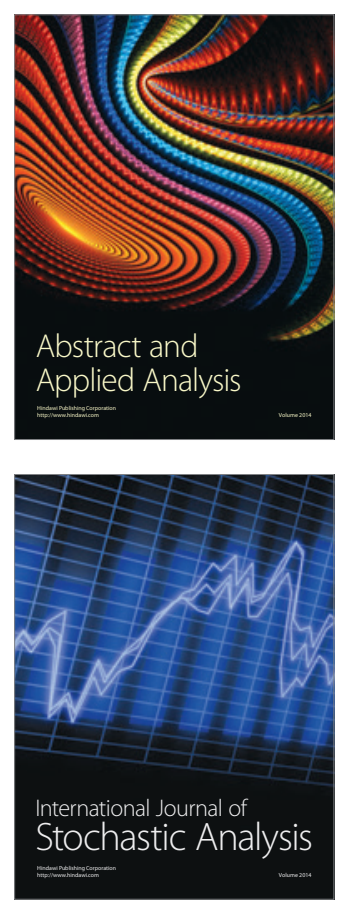

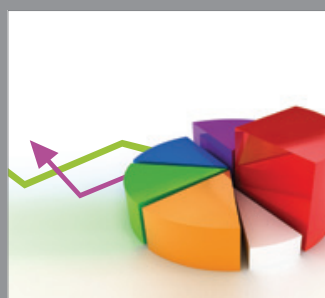

ournal of

Probability and Statistics

Promensencen
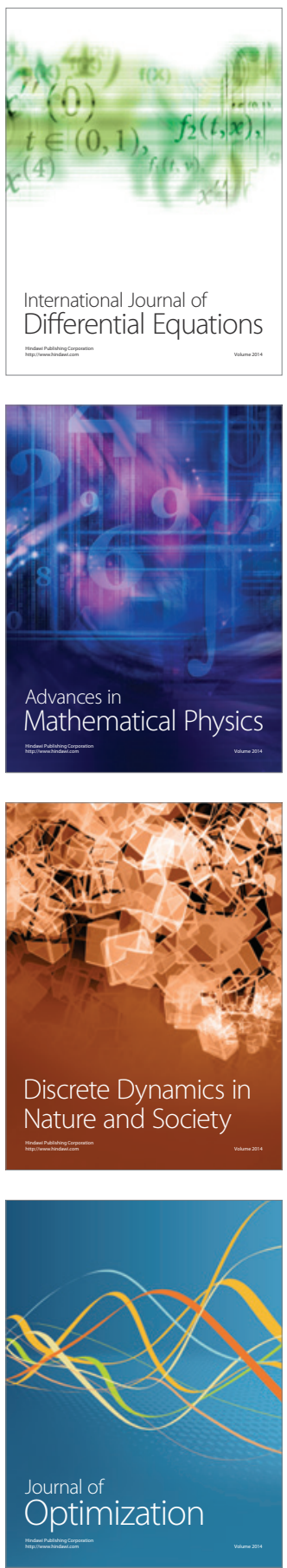